Pacific Journal of Mathematics

ATON OF NORMED RIM 


\title{
A GENERALIZATION OF NORMED RINGS
}

\author{
RICHARD ARENS
}

1. Introduction. A normed ring is, as is well known, a linear algebra $A$ over the complex numbers or the real numbers with a norm having, besides the usual properties of a norm, also the "ring" property

1.1.

$$
\|x y\| \leq\|x\|\|y\|
$$

The generalization studied here is that instead of merely one norm defined on $A$ there is a family of them, each satisfying 1.1; but of course it is natural to permit $\|x\|=0$ even though $x \neq 0$, to which attention is drawn by prefixing the word 'pseudo.'

The theory can be briefly summed up by saying that a pseudo-ring-normed algebra $A$ is an "inverse limit" of normed algebras. The main tool, which is rather obvious, is the fact that for a given pseudo-norm $V$ (we avoid the use of the double bars since an additional symbol would still be needed to distinguish the various pseudo-norms) those $x$ for which $V(x)=0$ form a two-sided ideal $Z_{V}$, and that $V$ can be used to define a norm in $A / Z_{V}$. When $A$ is complete some questions, such as whether $x$ has an inverse, can be reduced to the corresponding question for the completion $B_{V}$ of $A / Z_{V}$. It is of course profitable to be able to reduce questions to $B_{V}$ because $B_{V}$ is a Banach algebra, while $A / Z_{V}$ need not be complete. However, it seems to be difficult to say in general what questions can be so reduced to the case of Banach algebras. (We have spent much time vainly trying to discover whether the question of an element's having a right inverse in $A$ is reducible to the same question for the various $B_{V}$.)

When a pseudo-ring-normed algebra $A$ has a unit, then the latter may not be an interior point of the set of regular elements, but inversion is nevertheless continuous on the set of regular elements. On the other hand, there are many dense proper ideals. We devote some time to the topologization of the space of maximal, nondense (and hence closed), left ideals. From this a "structure space" of the topologically significant primitive ideals can easily be obtained, although we do not pursue the latter topic. 
In the commutative case, for each $\mathcal{L}$ in $L$ and $x$ in $A$, one can define $x(\mathcal{L})$ to be a complex number, just as for the normed case. There is given a sufficient condition that the $x($ ) be continuous, and this leads directly to be a characterization, among pseudo-ring-normed linear algebras over the complex numbers, of the space of continuous functions on a locally compact paracompact Hausdorff space.

Except for the part having to do with the paracompactness, which depends on the existence of a "locally-finite partition of unity," this paper was presented to the American Mathematical Society in November, 1946 (Bull. Amer. Math. Soc. Abstract 53-1-93). A forthcoming memoir of the American Mathematical Society, being prepared independently by Dr. Ernest Michael [16], on the subject of generalizations of normed rings, will treat many of these topics in greater detail.

2. Pseudo-valuations and pseudo-norms. A pseudo-valuation in a ring $A$ is a nonnegative real-valued function $V$ satisfying

$$
V(x+y) \leq V(x)+V(y), V(x y) \leq V(x) V(y), V(-x)=V(x), V(0)=0 .
$$

If $A$ is a linear algebra over the field $K$ (the real or complex numbers), and we have

$$
V(\lambda x)=|\lambda| V(x)
$$

as well as the other properties, we call $V$ a pseudo-ring-norm. In a topological ring we shall call a pseudo-valuation continuous if the set on which $V(x)<e$ is open for each real $e$.

We shall call a ring $A$ pseudo-valuated if there is a family $U$ of pseudovaluations such that $V(x)=0$ for all $V$ in $V$ only if $x=0$. It is not hard to see that $A$ becomes a topological ring if the various translations of the sets on which $V(x)<e$, where $e$ is real and $V$ ranges through $U$, are taken as a subbase [14] for open sets. A pseudo-valuated $\operatorname{ring} A$ is called complete when it is complete with respect to the uniform structure defined by the various relations $V(x-y)<e$.

2.1. THEOREM. Let $U$ be a neighborhood of 0 in a topological linear algebra $A$. Then there is a continuous pseudo-ring-norm, $V$, such that $U$ contains the set on which $V(x)<1$ if and only if $U$ is convex and $U U$ lies in $U$.

We leave the proof to the reader, except that we give a formula for $V$ when $U$ is given:

$$
V(x)=\sup _{|\lambda|=1} \inf \{r ; r \geq 0, x \in \lambda r U\}
$$


3. Quasi-inversion. In a ring $A, y$ is a right quasi-inverse of an element $x$ if

$$
x+y+x y=0
$$

[cf. 12, 11], and $x$ is a left quasi-inverse of $y$.

The methods of normed rings can be adapted to establish the following continuity property of quasi-inversion.

3.1. THEOREM. Let $R$ be the set of elements having right quasi-inverses in a pseudo-valuated ring A. Let $y$ be a left quasi-inverse of a limit point $z$ of $R$. For each $x$ in $R$, select a right quasi-inverse $x^{\prime \prime}$ of $x$. Then

3.2 .

$$
y=\lim _{x \rightarrow z, x \in R} x^{\prime} .
$$

Proof. The expressions to be written down will seem to involve the assumption that $A$ has a unity element 1 . However, such equations as we shall write down can always be freed of this assumption by expansion and cancellation.

Now by hypothesis we have,

$$
(1+y)(1+z)=1 \text { and }(1+x)\left(1+x^{\prime}\right)=1
$$

for $x$ in $R$. Let $u=z-x$ and $v=x^{\prime}-y$. Then

$$
(1+z+u)(1+y+v)=1 .
$$

Multiplying this on the left by $1+y$ we obtain

3.3.

$$
v=(1+y) u v+(1+y) \mathrm{u}(1+y) .
$$

Let $V$ be a pseudo-valuation. Since

$$
V((1+y) u)=V(u+y u) \leq V(u)+V(y) V(u)=(1+V(y)) V(u),
$$

we get

$$
V(u) \leq(1+V(y)) V(u) V(v)+(1+V(y))^{2} V(u) .
$$

If $V(u) \longrightarrow 0$, then presently

$$
V(u)<(1+V(y))^{-1},
$$

and then 


$$
V(v) \leq(1+V(y))^{2} V(u)(1-(1+V(y)) V(u))^{-1} ;
$$

this shows that $V(v) \rightarrow 0$.

Since, in $3.1,1+x$ tends to $1+z$ and $1+x^{\prime}$ tends to $1+y$, the continuity of multiplication shows that $(1+x)\left(1+x^{\prime}\right)$ tends to $(1+z)(1+y)$, so that $y$ is also a right quasi-inverse of $z$. For the sake of clarity we reformulate this result for the special case of a pseudo-valuated ring with unity element.

3.4. COROLLARY. If $z$ is a left regular limit of right regular elements, then $z$ is also right regular, and right inversion is continuous at $z$.

A topological ring in which inversion is not continuous at 1 , and which is ( consequently) not pseudo-valuated, is $L^{\omega}[2, \mathrm{p} .629]$.

4. Expansions for quasi-inverses. With the hypotheses of 3.1 , not only is right quasi-inversion continuous at $z$, it is analytic, in a sense which we shall not further define.

4.1. THEOREM. Let the hypothesis and notation of 3.2 be assumed. For each $x$ in $R$ and each $n$, let

$$
y_{n}(x)=\sum_{i=0}^{n}((1+y)(z-x))^{i}(1+y)-1 .
$$

Then for any pseudo-valuation $V$ and any $x$ in $R$ such that

$$
V(x-z)<(1+V(y))^{-1},
$$

we have

$$
\lim _{n \rightarrow \infty} V\left(y_{n}(x)-x^{\prime}\right)=0
$$

Proof. Using $u$ and $v$ as in the proof of 3.2 , we rewrite 3.3 as

$$
(1-(1+y) u)\left(1+x^{\prime}\right)=1+y \text {. }
$$

Let $v_{n}=y_{n}(x)-x^{\prime}$. Substituting here the expansion for $y_{n}(x)$, multiplying by $(1-(1+y) u)$ on the left, and using 4.2 , we obtain

$$
v_{n}=(1+y) u v_{n}-((1+y) u)^{n+1}(1+y) .
$$




$$
V(u) \leq r<(1+V(y))^{-1} \text {, }
$$

it follows readily that

$$
(1-r) V\left(v_{n}\right) \leq(1+V(y))^{n+2} V(u)^{n+1},
$$

from which the conclusion follows.

REMARK. The infinite series obtained by setting $n=\infty$ in $y_{n}$ is of no use in showing the existence of right-quasi-inverses even when $A$ is complete, as is done in the theory of Banach algebras. The reason is that a formal power product series in $A$ has something like a radius of convergence for each $V$, and if these are not bounded away from 0 then the series may not converge in $A$.

5. Direct operators. Let $L$ be an abelian group, and suppose there are defined in $L$ a number of real-valued functions $P$ such that

$$
P(x) \geq 0, P(0)=0, P(x-y) \leq P(x)+P(y)
$$

A special case of this are the "pseudo-norms" of convex topological linear spaces [cf. 15]. Let $P$ be any set of such $P$ 's defined in $L$. Then an endomorphism $\alpha$ of $L$ into itself will be called $p$-direct if for every $P$ in $P$ and each positive $e$ there is a positive $d$ such that $P(x)<d$ implies $P(\alpha x)<e$. The implication of this requirement evidently depends on the size of the family $P$. For example, if $L$ is a convex topological linear space, and $P$ is the class of all continuous pseudo-norms in $L$, then a $p$-direct linear operator in $L$ is necessarily a scalar multiple of the identity.

There is another application of the idea of direct operators which we mention in passing. Let $L$ be a Banach space, and let $\varepsilon$ be a Boolean ring (with unit) of projections in $L$. For $E$ in $\varepsilon$, we can define a pseudo-ring-norm $P_{E}$ by

$$
P_{E}(x)=\|E x\|
$$

The result we wish to state is the following.

5.1. THE OREM. A bounded operator $\alpha$ in $L$ is direct with respect to the pseudo-norms $P_{E}$ if and only if $\alpha E=E \alpha$ for all $E$ in $\varepsilon$.

Proof. For each $E$ in $\varepsilon$, we have

$$
\|E \alpha x\| \leq C_{E}\|E x\|
$$


Now let $x=(1-E) y$. Then

$$
\|E \alpha(1-E) y\| \leq C_{E}\|E(1-E) y\|=0
$$

for all $y$, or $E \alpha=E \alpha E$. Similarly $(1-E) \alpha=(1-E) \alpha(1-E)$. Expanding this and comparing with the former yields $E \alpha=\alpha E$, as desired.

Continuing with the general discussion, let us suppose that $L$ is a linear space, and that $P$ is a fixed family of pseudo-norms. Let $D p(L)$, or more briefly

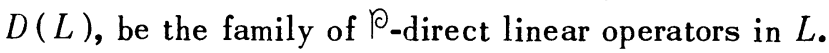

5.2. THEOREM. The family $D(L)$ is a linear algebra with unit element, and the $V_{P}$, where

$$
V_{P}(\alpha)=\sup _{P(x) \leq 1} P(\alpha x)
$$

form pseudo-ring-norms for $D(L)$.

We shall omit the proof, which is easy.

For our purposes, a linear space $L$ with a family $P$ of pseudo-norms $P$ shall be called complete if

a) $P(x)=0$ for every $P$ in $P$ implies $x=0$ and

b) whenever $P\left(x_{\mu}-x_{\nu}\right)$ converges to 0 for some directed set $x_{\mu}$ in $L$, and every $P$ in $P$, there is an $x$ in $L$ such that $P\left(x_{\mu}-x\right)$ converges to 0 for every $P$.

This definition obviously applies to ring-pseudo-normed linear algebras. Concerning $D(L)$ we may assert the following, again leaving the proof to the reader.

5.3. THEOREM. If $L$ is complete (with respect to the pseudo-norms $P$ in $P)$, then $D(L)$ is complete with respect to the $V_{P}$.

The purpose of the preceding discussion is to make possible the following statement.

5.4. THEOREM. Let $A$ be a linear algebra with unit element and a family $U$ of pseudo-ring-norms such that $V(1)=1$ for each $V$ in $U$. Then $A$ is isomorphic, with preservation of pseudo-norms, to a subalgebra of $D(B)$, where $B$ is $A$ regarded as a pseudo-normed linear space with family $U$ of distinguished pseudo-norms.

For the proof of 5.4 we represent each $x$ in $A$ by the operator that sends $y$ into $x y$. 
6. Completeness of kernel quotients. Let $A$ be a ring with a family $U$ of pseudo-valuations, and suppose $V_{1}, \ldots, V_{n}$ belong to $U$. Then

$$
V(x) \equiv \max \left(V_{1}(x), \cdots, V_{n}(x)\right)
$$

defines a pseudo-valuation in $A$. Those $x$ with $V(x)=0$ form a two-sided ideal $Z_{V}$, a kernel ideal of $A$ (with respect to $U$ ). We could have limited our attention to the case $n=1$ by assuming that $V \in U$ whenever $V_{1}, \ldots, V_{n} \in U$, but it is convenient not to assume this. The quotient ring $A_{V}=A / Z_{V}$ is a kernel quotient, and $V$ may be defined in it in anvious way.

When $V$ is a pseudo-ring-norm, $A_{V}$ is a normed ring.

The canonical homomorphism of $A$ onto $A_{V}$ is continuous when the topology described in $\$ 2$ is used in $A$, and that defined by $V$ is used in $A_{V}$. The completion in that topology of $A_{V}$ will be denoted by $\overline{A_{V}}$ and called a completed kernel quotient. In the ring-pseudo-norm case, the completed kernel quotients are all Banach algebras.

We shall now give several examples to show that we have no right to suppose that $A_{V}$ is complete even when $A$ is. In these examples, which are algebras $A$ of complex-valued continuous functions $f$ on various spaces $X$, we presuppose pseudo-ring-norms of the following type. Let $\mathcal{Z}$ be a class of compact sets whose interiors cover $X$. For each $K$ in $\alpha$ let $V_{K}(f)$ be the maximum of $|f(t)|$ for $t$ in $K$ (the topology in $A$ is then the $k$-topology, and $X$ necessarily is locally compact).

6.1. THEOREM. Let $T$ be completely regular, and let $C(T)$ be the ring of continuous functions on $T$. Then $C(T)$ is complete, and each $C(T)_{V_{K}}$ is complete.

6.2. THEOREM. Let $H(D)$ be the holomorphic functions on an open set $D$ in the plane. Then $H(D)$ is complete; but if $K$ has at least one limit point, then $H(D)_{V_{K}}$ is not complete.

6.3. THEOREM. Let $B C\left(R_{1}\right)$ be the ring of bounded continuous functions on the real line. Then $B C\left(R_{1}\right)$ is not complete, but each $B C\left(R_{1}\right)_{V_{K}}$ is complete.

In 6.1, $C(T)$ is well-known to be complete [1]; $Z_{V_{K}}$ consists of those functions which vanish on $K$, and so $C(T)_{V_{K}}$ is naturally isomorphic to a subalgebra of $C(K)$. By consideration of the Stone-Čech compactification, or otherwise, one can extend any function continuous on $K$ to all of $T$ (as a matter of fact, without increase of bound). Hence the subalgebra in question is all of $C(K)$ which is complete in its norm. 
Morera's theorem shows that $H(D)$ is closed in $C(D)$, and so it is complete. Again $I(D)_{V_{K}}$ is isomorphic to a subalgebra $S$ of $C(K)$. (Of course $Z_{K}$ contains only 0.) This subalgebra is not closed, however, for we can select an analytic function $f$ holomorphic on $K$ but with a singularity somewhere on $D$. It is the uniform limit of polynomials on $K$ and hence a limit of $S$ in $C(K)$, whence $S$ is not closed. We emphasize that this example shows that $Z_{V}$ may be 0 alone, while the topology of $A_{V}$ is not the same as that of $A$.

Finally, $B C\left(R_{1}\right)$ is not complete since it is dense in $C\left(R_{1}\right)$; but each $B C\left(R_{1}\right)_{V_{K}}$ is complete for the very reasons given for 6.1 .

7. Right inverses. In a later section we want to show that each maximal ideal of a commutative complete pseudo-valuated ring $A$ is a "divisor" of some kernel ideal $Z_{V}$. The following theorem together with Gelfand's principle yields this result. It obviously implies that, if $A$ is complete, an element $x$ which has a (two-sided) quasi-inverse in each $\overline{A_{V}}$ (the completion of $A_{V}$ ) has a quasiinverse in $A$, since a two-sided quasi-inverse is a unique right quasi-inverse. We have not been able to drop the requirement of "unique" in 7.1, since there seem to be difficulties in combining the various right inverses which are supposed to exist. If it should be in fact impossible to prove 7.1 without the word "unique," then this would be the first indication of a serious divergence between the theory of pseudo-valuated rings and that of normed rings, after which it is patterned.

After Theorem 7.1, we present a theorem like 7.1 in which the word "unique" is omitted, but there are other hypotheses which are by no means always fulfilled.

7.1. TH ЕОRЕм. Let $x$ be an element of a complete pseudo-valuated ring $A$. Then $x$ has a unique right quasi-inverse in $A$ if and only if its image in each completed kernel quotient has a unique right quasi-inverse there.

Proof: There is no loss in generality here in supposing the class $U$ of pseudo-valuations to contain

$$
V(x) \equiv \max \left(V_{1}(x), \cdots, V_{n}(x)\right)
$$

when it contains $V_{1}, \ldots, V_{n}$. Let $X_{V}$ be the image of $x$ in the kernel quotient $A_{V}$, and let $Y_{V}$ be the right quasi-inverse of $X_{V}$ in $\overline{A_{V}}$. For each positive integer $n$, one can find an element $y_{V, n}$ in $A$ such that its image $Y_{V, n}$ in $\bar{A}_{V}$ is close to $Y_{V}$ :

$$
V\left(Y_{V, n}-Y_{V}\right)<1 / n
$$


The index-pairs $V, n$ on these $y_{V, n}$ may be partially ordered by setting $(U, m) \leq$ $(V, n)$ whenever $m \leq n$, and $U(z) \leq V(z)$ for all $z$ in $A$ (this latter we abbreviate $U \leq V)$. When $U \leq V$, we have $Z_{V} \subset Z_{U}$; moreover we have a natural mapping (of bound 1) of $\overline{\bar{A}}_{V}$ into $\bar{A}_{U}$, and hence we can act as if an element originally introduced as belonging to $\bar{A}_{V}$ ( such as $Y_{V}$ ) also belongs to $\bar{A}_{U}$. As a matter of fact, with this convention, we have $Y_{V}=Y_{U}$ when $U \leq V$ because $Y_{V}$ is clearly a right quasi-inverse of $X_{U}$ in $\bar{A}_{U}$, and this was supposed to be unique. Making use of this fact, we shall show that $\left\{y_{V, n}\right\}$ forms a Cauchy system. Let $U$ belong to $U$, and suppose $V, \mathbb{W} \geq U$. Then

$$
\begin{aligned}
U\left(y_{V, m}-y_{W, n}\right) & =U\left(Y_{V, m}-Y_{W, n}\right) \\
& \leq U\left(Y_{V, m}-Y_{U}\right)+U\left(Y_{U}-Y_{W, n}\right) \\
& \leq \frac{1}{m}+\frac{1}{n} .
\end{aligned}
$$

Because of the assumption made at the outset about max $\left(V_{1}, V_{2}\right)$ belonging to $U$ with $V_{1}$ and $V_{2}$, the indices form a directed set; and the $y_{V, n}$ form a Cauchy directed system, which must converge to a $y$ in $A$ since $A$ is complete. A calculation similar to that just performed shows that

$$
V(x y+x+y)=0
$$

for all $V$, whence $y$ is a right quasi-inverse for $x$, as desired. This proves 7.l.

Let $A$ be a pseudo-valuated ring, and suppose that for each $V$ in $V$ there is selected an element $u_{V}$ of $A$ such that

7.2. for each $W$ in $U$ there is a finite set $U_{W}$ such that $W\left(u_{V}\right) \neq 0$ only for those $V$ which belong to $U_{W}$, and $W\left(u_{V}\right) \leq 1$;

7.3. $W\left(y-\sum_{y u_{V}}\right)=0$, the sum being extended over all $V$ in $U_{W}$;

7.4. for a fixed $V$, we have $\mathbb{W}\left(u_{V}\right) \neq 0$ only when $W$ belongs to $U_{V}$.

Then we shall call $u_{V}$ a locally finite partition of unity.

The partial sums of the series $\sum_{u_{V}}$ clearly form a Cauchy system, so that when $A$ is complete the existence of a locally finite partition of unity ensures the existence of a unity element, and makes it possible to talk about inverses rather than quasi-inverses.

We can exhibit nontrivial examples of such partitions.

7.5. THEOREM. Let $C(X)$ be the ring of continuous complex-valued functions on a locally compact, paracompact [ see 8] Hausdorff space T. Then a 
family $P$ of pseudo-norms can be defined in $C(T)$ so that the ring $D(C(T))$ of direct operators in $C(T)$ pseudo-normed as in 5.2, possesses a locally finite partition of unity, and is complete.

Proof. According to the hypothesis we can obtain a neighborhood-finite family $\{G\}$ of open sets which cover $X$ and whose closures are compact.

Using Theorem 6 of [8] and the method of Bourbaki (partition continue de l'unité) we construct a family of continuous real-valued nonnegative functions $f_{G}$, where $f_{G}(t) \neq 0$ only for $x$ in $G$ and $\sum f_{G}(t)=1$. As pseudo-norms in $C(T)$, take

$$
P_{G}(f)=\sup _{t \in G}|f(t)|
$$

The topology thus obtained is the $k$-topology, in which $C(T)$ is complete, and hence $D(C(T))$ is complete. The operators $u_{V}$ defined by $u_{V}(f)=f_{G} f$, where $V=V_{P_{G}}$ (see 5.2), are surely direct. Now let $G, H$ belong to $\{G\}$, and let $V, \mathbb{W}$ be $V_{P_{G}}, V_{P_{H}}$ respectively. Then $W\left(u_{V}\right) \neq 0$ only if $H$ is one of the finitely many sets of $\{G\}$ which meet the compact closure of $G$, and only if $G$ is one of the finitely many sets of $G$ which meet the closure of $H$. Except for details, this proves 7.5 .

7.6. THEOREM. Let $x$ be an element of a complete pseudo-valuated ring A possessing a locally finite partition of unity. Then $x$ has a right inverse in $A$ if and only if its image in each completed kernel quotient has a right inverse there.

Proof. We adopt the notation of 7.2-7.4. We do not suppose that the class $U$ here is closed under the maximum formation mentioned in the proof of 7.1, because this would require a complicated reformulation of 7.4. For each $V$ in $U$, define $V_{1}(z)=\max W(z)$ for all $W$ in $U_{V}$, and suppose $V$ itself to be adjoined to $V_{V}$. Let $X_{V_{1}}$ be the image of $x$ in $\bar{A}_{V_{1}}$, and let $Y_{V}$ be a right inverse of $X_{V_{1}}$ in $\bar{A}_{V_{1}}$. Select $y_{V, n}$ in $A$ so that if $Y_{V, n}$ is its image in $\bar{A}_{V_{1}}$, then for $n=1,2, \cdots$, we have

7.7.

$$
\left(V_{1}(x)+1\right) V_{1}\left(Y_{V, n}-Y_{V}\right)<2^{-n-1} \text {. }
$$

By the local finiteness, $y_{n}=\sum_{V} y_{V, n} u_{V}$ converges. Let $W$ belong to $V_{V}$. Then

$$
W\left(y_{n+1}-y_{n}\right) \leq \sum W\left(y_{V, n+1}-y_{V, n}\right) W\left(u_{V}\right) \text {. }
$$

Since this sum needs to be extended only over the $V$ in $U_{W}$, and since then $W$ 
lies in $U_{V}$ so that $W \leq V_{1}$, we have from 7.7 that the left member is less than $2^{-n}$ times the number of elements in $V_{W}$. Hence the sequence of $y_{n}$ is Cauchy and converges to some $y$ in $A$. Now

$$
W(x y-1)=\lim W\left(x y_{n}-1\right),
$$

and

$$
x y_{n}-1=\sum\left(x y_{V, n}-1\right) u_{V},
$$

so that

7.8 .

$$
W\left(x y_{n}-1\right) \leq \sum \mathbb{W}\left(x y_{V, n}-1\right),
$$

where this sum needs to be extended only over the $V$ in $V_{W}$. But then in each case $W$ belongs to $U_{V}$, and so $W \leq V_{1}$ and

$$
\begin{aligned}
W\left(x y_{V, n}-1\right) & \leq V_{1}\left(x y_{V, n}-1\right)=V_{1}\left(x_{V_{1}}, Y_{V, n}-X_{V_{1}} Y_{V}\right) \\
& \leq V_{1}(x) V_{1}\left(Y_{V, n}-Y_{V}\right)<2^{-n-1} .
\end{aligned}
$$

Since 7.8 involves only a fixed finite number of terms of this sort, we conclude that $W\left(x y_{n}-1\right)$ tends to 0 , whence $x y=1$ as desired.

8. Ideals. In topological rings, naturally the closed ideals play a more important part than the others. Much of the success of Banach algebras is due to the fact that maximal (that is, maximally proper) ideals are closed. The same is true for pseudo-valuated rings with only a finite set of pseudo-valuations. However, it is not true in general. For example, if in the case of the ring in 6.1, when $T$ is not compact, the ideal of functions each vanishing outside some compact set is swelled (by Zorn's lemma) to a maximal ideal $M$, then this ideal is certainly not closed. For every closed ideal in $C(T)$ can easily be shown to consist of all functions vanishing at a suitable point of $T$, and for each $t$ of $T$ there is an $f$ in $M$ which is not zero there. This ideal is consequently dense in $C(T)$.

Our idea is to reduce certain parts of the ideal theory (and at some future time, of the representation theory) of pseudo-valuated rings to that of the completed kernel quotients, in which some of the techniques of Banach algebras can be applied. For terms used below but not defined, see [11].

8.1. THEOREM. In a pseudo-valuated ring, every nondense left and/or regular and/or two-sided ideal $L$ is contained in a closed left and/or regular 
and/or two-sided ideal $N$ which contains a kernel ideal.

Proof. Let $L$ be a nondense ideal. Then there is a $u$ in $A$, a $V$ in $V$, and a positive $e$, such that $V(u-x)<e$ implies $x \notin L$. Let $M$ be the sum of $L$ and the kernel ideal $Z_{V}$. Let $x \in M$. Then $x=y+z, y \in L$, and $z \in Z_{V}$. Now

$$
e \leq V(u-y)=V(u-x+z) \leq V(u-x),
$$

whence $u$ does not belong to the closure $N$ of $M$. Hence $N$ is the desired ideal. However, if $L$ is regular with relative right unit $v$, we must show that there is a relative right unit modulo $N$. But $v$ itself can obviously be chosen for this purpose.

8.2. COROLLARY. Every nondense maximal (two-sided) or maximal left ideal is closed and contains a kernel ideal.

9. An abstract approach to structure spaces. We note that the ideas of Stone and Jacobson can be generalized to topologizing suitable subsets of partially ordered systems. In what follows one may think of $k$ as the class of ideals of a ring, $\mathcal{J}$, the two-sided ideals, and $\mathcal{L}$, the maximal left ideals.

Let $\&$ be a partially ordered set, $\mathfrak{J}$ a subset of $\&$ which forms a complete lattice with greatest element $A$, and $\mathcal{L}$ an arbitrary subset of $\ell$. We want to define a closure operation in $\mathcal{L}$. For $\Re \subset \mathcal{L}$, let

$$
i(\mathfrak{M})=\sup \{a ; a \in \mathfrak{J}, a \leq \mathfrak{l} \text { for every } \mathfrak{l} \text { in } \mathbb{M}\}
$$

For $a$ in $\mathfrak{g}$, let

$$
\mathfrak{H}(a)=\{\mathfrak{l} ; \mathfrak{l} \in \mathcal{L}, \mathfrak{l} \geq a\}
$$

One can easily verify that

9.1 .

$$
u\left(\sup a_{\alpha}\right)=\Pi u\left(a_{a}\right) .
$$

For $\mathbb{M} \subset \mathcal{L}$, the closure $\overline{\mathbb{M}}$ shall be $\mathfrak{i}(\mathfrak{M})$.

The next three propositions, whose proofs are omitted, mention all the closure axioms needed for a topological $(T-)$ space; $\Lambda$ is the void set: $\bar{\Lambda}=\Lambda$ if and only if $A$ has no upper bound in $\mathcal{L}$.

9.3. $\mathbb{M} \subset \bar{M} ; \mathfrak{M} \subset \Re$ implies $\bar{M} \subset \overline{\mathscr{N}}$; and $\overline{\mathscr{M}}=\overline{\mathscr{M}}$.

9.4. $\overline{(M \cup \Re)} \subset \bar{M} \cup \bar{\Re}$ holds generally in $\mathcal{L}$ provided for each $\mathfrak{l}$ in $\mathcal{L}$, the inequality $\mathfrak{l} \geq a \wedge \mathfrak{b}$ implies $\mathfrak{L} \geq a$ or $\mathfrak{l} \geq \mathfrak{b}$. 
9.5. THEOREM. If the conditions of 9.4 and 9.2 are satisfied, $\mathcal{L}$ becomes a $T$-space.

As remarked by Jacobson, $\mathcal{L}$ need not be a $T_{1}$-space, nor a $T_{2}$-space even when it is a $T_{1}$-space. As a matter of fact, it need not even be a $T_{0}$-space, but this defect is smaller than the other two, for it may be "removed" by a process of identification.

These spaces $\mathcal{L}$ often have compactness properties.

9.6. THEOREM. The set $\mathfrak{u}(a)$ is compact if and only if given $a_{a}$ in J with $a_{a} \geq a$ such that every finite collection has an upper bound in $\mathcal{L}$, then the entire collection has an upper bound in $\mathcal{L}$.

10. Application to rings. Now let $A$ be a ring and let $g$ be the class of trosided ideals. Let $\mathcal{L}$ be a class of ideals of $A$ each of which is either

10.1. prime and proper,

10.2. maximal left, or

10.3. primitive.

Then the condition of 9.4 holds ( case 10.3 was considered by Jacobson, and the others are obvious), and $\mathcal{L}$ becomes a space of 9.5 .

The special case in which $\mathcal{L}$ consists of the maximal left ideals is interesting. It is fairly easy to see that $i(\{\mathfrak{L}\})$ is always primitive and that we thus obtain an open continuous mapping on Jacobson's structure space.

In topological rings, $\mathcal{L}$ may be chosen to contain only closed ideals, while $\exists$ may be chosen as before. This does not adversely affect the fulfillment of the closure axioms, but naturally when the class of ideals in $\mathcal{L}$ is restricted, limit points are lost and compactness is affected. Indeed, even when $A$ is commutative and has a unit, $\mathcal{L}$ may be noncompact (see 8.1 ). Hence we prove the following about pseudo-valuated rings.

10.4. THEOREM. Let $\mathcal{L}$ be the class of nondense maximal left ideals, and I the two-sided ideals, of a pseudo-valuated ring A. Let a be a regular member of $\mathrm{J}$, and let $Z_{V}$ be a kernel ideal. Then $\mathfrak{u}(a) \cap \mathfrak{u}\left(Z_{V}\right)$ is compact.

Proof. Without loss of generality (see 9.1) we may suppose $a \supset Z_{V}$, and thus $\mathfrak{u}(a) \subset \mathfrak{u}\left(Z_{V}\right)$. Now let $a_{a} \supset a$. Suppose that for $\alpha_{1}, \ldots, \alpha_{n}$ there is an $\mathfrak{L}$ in $\mathcal{L}$ containing the $a_{a_{i}}$ for $i=1, \cdots, n$. Let $B=A / a$, pseudo-valuated by means of $V$. Since $a$ is regular, $B$ has a unit, and $\mathfrak{l}$ does not map onto $B$. The multiplicative properties of $V^{\prime}$ ensure that the closure of the image of $\mathfrak{l}$ is also 
an ideal in the completion $\bar{B}$. Thus the images of $a_{a_{1}}, \ldots, a_{a_{n}}$ generate a proper left ideal in $\bar{B}$. Hence, by the argument of Banach algebras (cf. [11]), the images of the $a_{a}$ all fall into one (closed) maximal left ideal of $\bar{B}$. The inverse image of this in $A$ provides a bound for the $a_{\alpha}$, and so 9.6 applies, finishing the proof.

As in an earlier section, we can go further with the assumption of a locally finite partition of unity.

10.5. Lemma. Let $\mathcal{L}, \mathcal{J}$, and $A$ be as in 10.4. Let $A$ have a locally finite partition of unity, $\left\{u_{V}\right\}$. Let $G_{V}$ be the (open) complement of $u\left(a_{V}\right)$, where $a_{V}$ is the two-sided ideal generated by $u_{V}$. Then $G_{V}$ does not meet $u\left(Z_{W^{\prime}}\right)$ for $W$ not in $V_{V}$.

Proof. Clearly $u_{V} \in Z_{\mathbb{W}}$ when $\mathbb{W} \in \cup_{V}$. Hence $a_{V} \subset Z_{\mathbb{W}}$, and so

$$
\mathfrak{u}\left(a_{V}\right) \supset \mathfrak{u}\left(Z_{W}\right),
$$

from which the conclusion follows.

10.6. ТнЕовем. Let $\mathcal{L}, \mathfrak{J}, A$, and $G_{V}$ be as in 10.5. Then each $G_{V}$ has a compact closure in $\mathcal{L}$, and the $G_{V}$ form a star-finite open covering of $\mathcal{L}$, which is consequently a paracompact ${ }^{*}$ locally compact space.

Proof. By 8.2 , the $u\left(Z_{V}\right)$ cover $\mathcal{L}$, and so $G_{V}$ must be contained in the union of those finitely many $u\left(Z_{W}\right)$ for which $W$ belongs to $U_{V}$, and this union is compact by 10.4. (Recall that $A$ has a unit.) Now suppose that $G_{V}$ and $G_{W}$ intersect in a nonvoid set. They must intersect in a point of some $u\left(Z_{U}\right)$, whence $U \in V_{V}, U \subset U_{W}$. For $V$ fixed, this rules out all but a finite set of possibilities for $W$. This shows that the $G_{V}$ form a star-finite system. Now let $\mathfrak{l}$ belong to $\mathcal{L}$. Then for some $V$, we have $u_{V} \notin \mathfrak{l}$; for otherwise we would have $1 \in \mathfrak{l}$ since the latter is closed. Then $\mathfrak{l} \in G_{V}$. Hence the $G_{V}$ cover $\mathcal{L}$, and $\mathcal{L}$ is therefore locally compact.

Now let an arbitrary open covering $C$ of $\mathcal{L}$ be given. For each $V$ select a finite number of these open sets to cover $G_{V}$, and cut these sets down so that they lie in the union of those $G_{W}$ which meet $G_{V}$. The class of sets so obtained is easily seen to form a neighborhood finite refinement of $C$. This completes the proof of 10.6 .

11. Characterization of the ring of continuous functions. We are now in a position to characterize the ring $A=C(T, K)$ ( $K$ is here the complex field),

* In the generalized sense obtained by removing the stipulation of Hausdorff separation from Dieudonné's definition. 
where $T$ is a locally compact, paracompact Hausdorff space, as a pseudo-ringnormed ring in which the topology is that of the $k$-topology. From 7.5 we say about $C(X, K)$ that

11.1. it has a locally finite partition of unity; and moreover, if $x^{*}$ is defined by $x^{*}(t)=\overline{x(t)}$, then

11.2. it has a semilinear operation $*$ such that $(\lambda x+y z)^{*}=\bar{\lambda} x^{*}+z^{*} y^{*}$, $x^{* *}=x$, and

11.3. $V\left(x x^{*}\right) \geq k_{V} V^{\prime}(x) V^{\prime}\left(x^{*}\right)$, where $k_{V}$ is some positive number, for each $V$ in $U$.

In $C(X, K)$, all the $k_{V}=1$.

The main theorem is a converse of these observations.

11.4. THEOR EM. A commutative complete pseudo-ring-normed linear algebra $A$ over the complex numbers $K$ satisfying $11.1,11.2,11.3$ is equivalent to a $C(T, K)$, where $T$ is a locally compact, paracompact llausdorff space which is homeomorphic to the space $\mathcal{L}$ of closed maximal ideals of $A$, topologized as in 10.6 .

Proof. Since each closed maximal ideal contains some $Z_{V}$, the corresponding residue class ring is a normed field, which must be the field of complex numbers $K$. For $\mathfrak{L}$ in $\mathcal{L}$, define $x(\mathfrak{L})=a$ if $x-a \cdot 1$ belongs to $\mathfrak{L}$. Now $A_{V}$ is isomorphic to a subset of $C\left(X_{V}, K\right)$ by Theorem 1 of [4], which is essentially the GelfandNeumark lemma. It follows that

11.5 .

$$
k_{V}^{2} I^{\prime}(x) \leq \sup _{\mathfrak{L} \in \mathcal{U}\left(Z_{V}\right)}|x(\mathfrak{l})| \leq V(x), x^{*}(\mathfrak{l})=\overline{x(\mathfrak{L})} .
$$

Let $p_{1}, p_{2}, \ldots$ be a sequence of ordinary polynomials with real coefficients such that

$$
\left|p_{m}(a)-\right| a||<2^{-m}
$$

for $a$ any real number with $|a| \leq m$. These can be constructed by Weierstrass' approximation theorem. It follows from 11.5 that if $x=x^{*}$ in $A$, then $p_{m}(x)$ is a Cauchy system, and for the limit y we surely have $|x(\mathfrak{l})|=y(\mathfrak{l})$ for each $\mathfrak{L}$ in $\mathcal{L}$. Denote this $y$ by $|x|$.

We must now establish that $x()$ is continuous on $\mathcal{L}$. Let $\mathfrak{C} \mathcal{L}$. Since $A$ has a unit we may suppose that $x(\mathfrak{L})=0$. We may also suppose that $x=x^{*}$. 
From the possibility of taking absolute values, it follows that

$$
y \equiv 1-|x|+|1-| x||
$$

belongs to $A$, and it has the value 2 at $\mathfrak{L}$. Now let a be the principal ideal generated by $y$, and suppose $m \in u(a)$. Then $y(m)=0$, so that $m \neq \mathfrak{L}$. Suppose that $|x(m)| \geq 1$ for some $m$ in $\mathcal{L}$. Then $y(m)=0$, or $m \in \mathfrak{u}(a)$. Hence the complement of $\mathfrak{u}(a)$ is a neighborhood of $\mathfrak{l}$ on which the absolute value of value of $x()$ is less than 1 . In view of the possibility of scalar multiplication, this shows that $x()$ is continuous.

We next show that the topology of $A$ is the same as the $k$-topology for the corresponding functions. If $\mathcal{W}$ is a compact subset of $\mathcal{L}$, it is contained in finitely many of the $G_{V}$ of 10.6, and by 10.5 it is contained in the union of some finite class of $U\left(Z_{V}\right)$ 's. Hence convergence in all pseudo-norms implies uniform convergence on $\mathcal{K}$ by 11.5 , hence in the $k$-topology. The other way around is simpler, depending on 11.5 and the fact that each $u\left(Z_{V}\right)$ is compact. An application of a generalized form [5, p. 765] of Kakutani's method for the StoneWeierstrass theorem completes the proof of 11.4.

One could go on to generalize the numerous variations of the Gelfand Neumark lemma involving only real scalars, or quaternions, and so on, but the method of reducing these questions to the corresponding case of normed rings is now clear. The purpose of the partition of unity is of course to enable one to disclose the topology of $A$ as the $k$-topology, and thus has no nontrivial counterpart in the "normed" theory. If that part of the previous proof which involves the $G_{V}$ is ignored, we obtain the following:

11.6. THEORЕM. A commutative complete pseudo-ring-normed linear algebra $A$, with unit over the complex numbers satisfying 11.2 and 11.3 , is isomorphic to a $C(T, K)$, where $T$ is a completely regular space homeomorphic to the space of closed maximal ideals, and such that the topology of $A$ corresponds to some topology in $C(T, K)$ which has at most the open sets of the $k$-topology.

This result, while perhaps more easily applicable, is not "a characterization of those $C(T, K)$ with a topology $t \leq k$," since such $C(T, K)$ do not need to be complete. In $[6$, p. 234] there is exhibited a space $T$ in which all compact sets are finite (although not always open as the next sentence in that paper should have said). Consequently, the completion of the space $C(T, K)$ in any topology $t$ which is $\leq k$ includes discontinuous functions.

When 11.3 does not hold, we have no way of knowing that the functions $x($ ) are continuous on $\mathcal{L}$; in fact, even in the norm case they are sometimes not 
continuous ( $c f$. Gelfand and Šilov). Of course, one can force them to be continuous by abandoning the topology in $\mathcal{L}$ and introducing a new one ad hoc, defining just enough sets to be open so that they are continuous. The result is a completely regular space, and in it we can make this statement:

11.7. THEовем. Let $A$ be a commutative complete pseudo-normed linear algebra over the complex or real numbers. For each $x$ in A, define a function $x()$ on the space of closed maximal regular ideals. Then $x$ has a quasi-inverse in $A$ if and only if $x(\mathfrak{L}) \neq-1$ for each $\mathfrak{L}$ in $\mathcal{L}$.

The proof follows from the preceding remarks, Theorem 7.1, and 8.2.

\section{REFERENCES}

1. Richard Arens, A topology for spaces of transformations, Ann. of Math. 47 (1946), $480-495$.

2. The space $L^{\omega}$ and convex topological rings, Bull. Amer. Math. Soc. 52 (1946), $931-935$.

3. $623-630$.

, Linear topological division algebras, Bull. Amer. Math. Soc. 53 (1947),

4. —, Representation of *algebras, Duke Math. J. 14(1947), 269-282.

5. - Approximation in, and representation of, certain Banach algebras, Amer. J. Math. 71 (1949), 763 - 790.

6. - Note on convergeñce in topology, Math. Mag. 23 (1950), 229-234.

7. N. Bourbaki, Elements de mathematique, VIII, Premiere parti, Livre III, Chapitre IX, Hermann et Cie., Paris, 1948.

8. J. Dieudonné, Une généralization des espaces compacts, J. Math. Pures Appl. 23 (1944), $65-76$.

9. I. Gelfand and M. Neumark, On the imbedding of normed rings into the ring of operators in Hilbert space, Rec. Math. (Mat. Sbornik) N.S. 12 (1943), 197-213.

10. _ and G. Ś Silov, Über verschiedene Methoden der Einführung der Topologie in die Menge der maximalen Ideale eines normierten Ringes, Rec. Math. (Mat. Sbornik) N.S. 9 (1941), $25-39$.

11. Einar Hille, Functional analysis and semi-groups, Amer. Math. Soc. Colloq. Publ. 31, New York, 1948.

12. N. Jacobson, The radical and semi-simplicity for arbitrary rings, Amer. J. Math. 47 (1945), $300-320$.

13. - A topology for the set of primitive ideals in an arbitrary ring, Proc. Nat. Acad. Sci. U.S.A. 31 (1945), 333- 338.

14. S. Lefschetz, Algebraic topology, Amer. Math. Soc. Colloq. Publ. 27 (1942), New York.

15. G. W. Mackey, On convex topological linear spaces, Trans. Amer. Math. Soc. 60 (1946), 519-537.

16. Ernest Michael, Locally multiplicatively-convex topological algebras, to appear in Mem. Amer. Math. Soc. 



\section{PACIFIC JOURNAL OF MATHEMATICS}

\section{EDITORS}

\section{R. M. RoBINSON \\ University of California Berkeley 4, California}

\author{
*R. P. Dilmorth \\ Califomia Institute of Technology \\ Pasadena 4, California
}

E. F. BeckenbaCh, Managing Editor

University of California

Los Angeles 24, California

${ }^{*}$ During the absence of Herbert Busemann in 1952.

\section{ASSOCIATE EDITORS}

$\begin{array}{llll}\text { R. P. DILWORTH } & \text { P. R. HALMOS } & \text { BØRGE JESSEN } & \text { J. J. STOKER } \\ \text { HERBERT FEDERER } & \text { HEINZ HOPF } & \text { PAUL LÉVY } & \text { E. G. STRAUS } \\ \text { MARSHALL HALL } & \text { R. D. JAMES } & \text { GEORGE PÓLYA } & \text { KÖSAKU YOSIDA }\end{array}$

\section{SPONSORS}

UNIVERSITY OF BRITISH COLUMBIA

CALIFORNIA INSTITUTE OF TECHNOLOGY

UNIVERSITY OF CALIFORNIA, BERKELEY

UNIVERSITY OF CALIFORNIA, DAVIS

UNIVERSITY OF CALIFORNIA, LOS ANGELES

UNIVERSITY OF CALIFORNIA, SANTA BARBARA

OREGON STATE COLLEGE

UNIVERSITY OF OREGON

\author{
UNIVERSITY OF SOUTHERN CALIFORNIA \\ STANFORD UNIVERSITY \\ WASHINGTON STATE COLLEGE \\ UNIVERSITY OF WASHINGTON \\ AMERICAN MATHEMATICAL SOCIETY \\ NATIONAL BUREAU OF STANDARDS, \\ INSTITUTE FOR NUMERICAL ANALYSIS
}

Mathematical papers intended for publication in the Pacific Journal of Miathematics should be typewritten (double spaced), and the author should keep a complete copy. Manuscripts may be sent to any of the editors. All other communications to the editors should be addressed to the managing editor, E. F. Beckenbach, at the address given above.

Authors are entitled to receive 100 free reprints of their published papers and may obtain additional copies at cost.

The Pacific Journal of Mathematics is published quarterly, in March, June, September, and December, by the University of California, Berkeley 4, California. The price per volume (4 numbers) is \$8.00; single issues, $\$ 2.50$. Special price to individual faculty members of supporting institutions and to individual members of the American Mathematical Society: $\$ 4.00$ per volume; single issues, $\$ 1.25$.

Subscriptions, orders for back numbers, and changes of address should be sent to the publishers, University of California Press, Berkeley 4, California.

Printed at Ann Arbor, Michigan. Entered as second class matter at the Post Office, Berkeley, California.

\section{UNIVERSITY OF CALIFORNIA PRESS • BERKELEY AND LOS ANGELES}




\section{Pacific Journal of Mathematics}

\section{Vol. 2, No. $4 \quad$ April, 1952}

Shmuel Agmon, On the singularities of Taylor series with reciprocal coefficients .................................... 431

Richard Arens, A generalization of normed rings ............... 455

Iacopo Barsotti, Intersection theory for cycles of an algebraic variety . . . . 473

Leonard M. Blumenthal, Two existence theorems for systems of linear inequalities ..................................... 523

Frank Herbert Brownell, III, Translation invariant measure over separable Hilbert space and other translation spaces................. 531

J. W. S. Cassels, On a paper of Niven and Zuckerman ............... 555

Nelson Dunford, Spectral theory. II. Resolutions of the identity .......... 559

Eugene Lukacs and Otto Szász, On analytic characteristic functions ...... 615

W. A. Mersman, Evaluation of an integral occurring in servomechanism theory.......................................... 627

Lawrence Edward Payne and Alexander Weinstein, Capacity, virtual mass, and generalized symmetrization......................... 633

Choy-Tak Taam, The boundedness of the solutions of a differential equation in the complex domain ...................................... 643 